sprayed daily with Gib, solution ( $20 \mathrm{mg} / 1$ ) in continuous days, in mixed regimes, while the sprayings on the control were applied every third day.

As shown in the table it is evident that for the Blanche de Pointin cultivar in short-day (control) the effect of Gib. was nil. On continuous day the plants treated with Gib. flowered with a delay of 15 days compared with the control on short day, and under the mixed regimes, on the variants 1 ( $1 \mathrm{sd} / 1 \mathrm{~cd})$ $3(2 \mathrm{sd} / 1 \mathrm{~cd})$ and $4(4 \mathrm{sd} / 2 \mathrm{~cd})$ where $\mathrm{Gib}$. has not been applied, the plants did not flower, while the treated ones did.

The Louis de Barthou cultivar, control with Gib. on shortday flowered 11 days earlier and the control with Gib. on continuous-day 11 days later, compared with the control in short-day. As to the mixed variants, the plants have flowered in the variant 3 , while the lowering was hastened in variants 1 a. 4. The number of the flowering plants has always been more numerous when Gib. sprayings were applied.

The results of these experiments have shown that the effect of Gib. was manifest under the mixed photoperiodic regimes and under the simple regimes as well; all these regimes being non-inductive by themselves.

The differences between the two chrysanthemum cultivars are only quantitative.

Centrul de Biologie, Cluj (dir. prof. E. POP)

$$
\text { I. BARBAT and C. OChEŞANU }
$$

Eingegangen am 8. Februar 1964

1) LANG, A.: Naturwissenschaften 43, 257, 284, 544 (1956). 2) Chovard, P.: Bull. soc. botan. France 105, 3-4 (1958). 3) Lang, A.: Fortschr. Botanik 22, 312-345 (1960).

\section{Zur Kinetik der Phytoalexinbildung}

Wie australische Forscher ${ }^{1 b}$ ), ${ }^{5}$ ) gezeigt haben, gehören die nach Infektion mit Sclerotinia fructicola bei Phaseolus vulgaris und Pisum sativum anfallenden Phytoalexine ${ }^{3}$ ): Phaseolin $\left(\mathrm{C}_{20} \mathrm{H}_{18} \mathrm{O}_{4}\right)$ bzw. Pisatin $\left(\mathrm{C}_{17} \mathrm{H}_{14} \mathrm{O}_{6}\right)$ der Stoffgruppe der Chromanocumarane an. Sie unterscheiden sich nur in der Konfiguration ihrer Substitutionsgruppen. Ferner fanden CRUICKSHANK und PerRin ${ }^{1 a}$ ), daB nach Infektion des Erbsen-Endokarps mit anderen potentiellen Parasiten (z.B. Erysiphe cichoracearum, Uromyces fabae oder Peronospora tabacina) ebenfalls Pisatin gebildet wird. Sogar anorganische Verbindungen, $\mathrm{CuCl}_{2}\left(3 \times 10^{-3} \mathrm{~mol}\right)$ und $\mathrm{HgCl}_{2}\left(3 \times 10^{-5}\right)$, lösen Pisatinbildung aus. Schon diese Befunde Iassen den Schluß $z u$, daß die chemische Konstitution der Phytoalexine lediglich von der stofflichen Zusammensetzung der infizierten Wirtszelle bestimmt wird. Somit käme den von dem Parasiten in die Wirtszelle entlassenen Stoffwechselprodukten nur die Funktion von Induktoren zu.

Zur Untermauerung dieser Auffassung bot sich die Isotopentechnik an. Als Versuchsobjekt diente das die Samenkammern von Phaseolus auskleidende Endokarp. Dieses wurde mit dem beim Steinobst hochvirulenten, bei Phaseolus jedoch avirulentèn Schlauchpilz Scleroinia fructicola beimpft. Zwei Versuchsreihen wurden angesetzt: Bei der einen (I) wurden Sporen benutzt, welche Reinkulturen entstammten, denen ${ }^{14} \mathrm{C}$-Glukose zugesetzt worden war; bei der anderen (II) war es das Wirtsgewebe, das eine ,Markierung's erfahren hatte. Als Kontrolle diente eine Versuchsserie (K), bei der das markierte Endokarp nur mit aqua bidest. beschickt wurde. Nach einer Inkubationszeit von 24 Std wurden die Roh-, Diffusate gesammelt und in der üblichen Weise durch Zentrifugieren und Ausschütteln mit Petroläther aufgearbeitet. Anschließend wurde der lipophile Anteil, der nahezu quantitativ die angefallenen Phytoalexinmengen enthält ${ }^{3 b}$ ), auf seine Radioaktivität geprüft.

Bei Versuchsreihe I war nur eine geringfïgige, offenbar innerhalb der Fehlergrenzen liegende Erhöhung (5\%) der Radioaktivität $\mathrm{zu}$ registrieren, im Unterschied $\mathrm{zu}$ dem hydrophilen Anteil, bei dem eine Steigerung auf etwa das 400 fache festzustellen war. Anders fielen die Ergebnisse bei Versuchsreihe II aus: Hier war bei der lipophilen, also der phytoalexin. haltigen Phase die Radioaktivität um nahezu $100 \%$ erhöht. Bei der Kontrollserie (K) belief sich die Steigerung auf nur $0,2 \%$.

Diese Ergebnisse entsprechen der Erwartung. Sie zeigen, daß Konstituenten der vom Parasiten in die Wirtszelle entlassenen Stoffwechselprodukte nicht in die Synthese der Phytoalexine eingehen und daher bei der Interaktion zwischen Wirt und Parasit nur die Rolle von Induktoren spielen können.
Eine solche Vorstellung steht in gutem Einklang mit der schon früher geäußerten Vermutung, daß die vitale Wirtszelle über einen mit dem Respirationssystem gekoppelten, aber nicht mit ihm identischen. Mechanismus verfügt ${ }^{2}$ ), welcher, einmal in Gang gesetzt, zur Entstehung jener Abwehrstoffe führt, die vor 25 Jahren postuliert und mit der Bezeichnung ,"Phytoalexine" belegt worden waren ${ }^{4}$ ). Die Frage, ob es sich hierbei lediglich um die Abspaltung einer Klein- aus einer präformierten Großmolekel oder um eine kompliziertere enzymatische Umsetzung innerhalb der Wirtszelle handelt, muß vorerst offen gelassen werden. Eine ausführliche Darstellung wird durch den erstgenannten Autor erfolgen.

Botanisches Institut der Universität, Nijmegen (Holland) G. VAN DEN ENDE a. K. O. MülleR

Eingegangen am 22. April 1964

1) Cruickshank, J.A.M., u. D.R. Perrin: a) Australian J. Biol. Sci. 16, 111 (1963); - - b) Life Sci. 2, 680 (1963). -2) Jerome, S.M.R. u. K.O. Müller: Australian J. Biol. Sci. 11, 301 (1958). — $\left.{ }^{3}\right)$ MüLLER, K.O.: a) Phytopathol. Z. 27, 237 (1956); - b) Australian J. Biol. Sci. 11, 275 (1958) - ${ }^{4}$ ) MÚLLER, K.O., u. H. BÖrGER: Arb. biol. Reichsanst. 23, 189 (1940). - 5) Perrin, D. R., u. W. BotтomLEY: J. Am. Chem. Soc. 84, 1919 (1962).

\section{Über đie Länge der septierten Fasern bei Tectona grandis}

Die gesetzmäßige Veränderung der Zellänge mit dem Alter im sekundären Xylem von Nadel- und Laubbäumen ist durch zahlreiche Untersuchungen weitgehend bekannt ${ }^{x}$ ). Die meisten Arbeiten galten der Länge von Tracheiden und Holzfasern. Die ontogenetische Entwicklung septierter Fasern wurde von GovindaraJalu und Swamy ${ }^{2}$ ) am Stammholz von Pithecolobium dulce BentH. geprüft. Hiernach sind diese über den Querschnitt hinweg stets länger als die nicht-septierten Fasern; sie zeigen ferner nicht den typischen Anstieg der Zelllänge mit zunehmendem Alter des Bildungsgewebes. CHow. DHURY et al. ${ }^{\mathbf{3}}$ ) fanden jedoch an Einzelproben einiger Angiospermen, daß je nach der Holzart die septierten Fasern länger oder kürzer als die nichtseptierten Fasern sein können.

Um weitere Unterlagen über die Entwicklung septierter Fasern zu erhalten, wurde eine Stammscheibe von Tectona

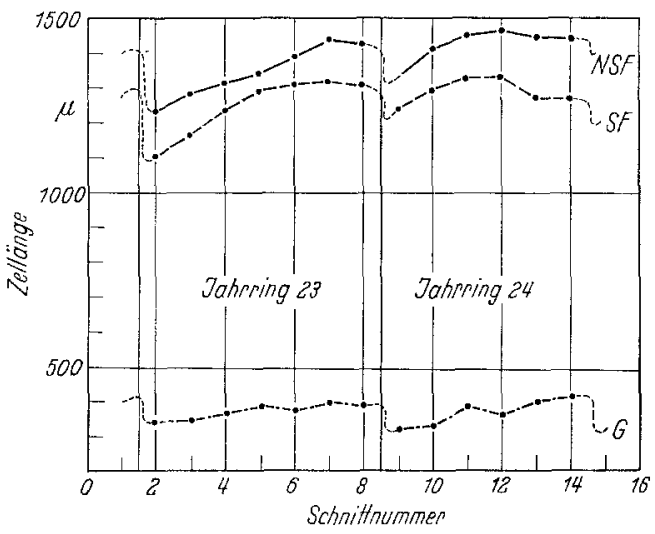

Fig. 1 Veränderung der Länge von Gefäßen (G), septierten (SF) und nicht-septierten (NSF) Holzfasern in zwei Jahrringen

grandis L. mit etwa $20 \mathrm{~cm}$ Durchmesser und 25 ringporigen Jahrringen untersucht. Die Faserlänge innerhalb eines Jahrringes wurde an zwei folgenden Jahrringen des Splintholzes mittels tangentialer Serienschnitte von etwa $400 \mu$ Stärke gemessen, die nach JEFFREY mazeriert worden waren. Um die Entwicklung der Faserlänge mit dem Alter zu erfassen, wurde an zwei einander gegenüberliegenden Radien die mittlere Zellänge von jedem 5. Jahrring untersucht. Zugleich wurden auch die GefäBe gemessen. Die in den Fig. 1 und 2 wiedergegebenen Mittelwerte wurden jeweils an 100 Fasern bzw. 50 Gefäßen ermittelt. In gleicher Weise wurde eine weitere Stammscheibe von Tectona grandis geprüft, die zerstreutporiges Holz aufwies.

Fig. 1 zeigt die Längenänderung der Zellarten in den beiden untersuchten Jahrringen. Die Länge der nicht-septierten und diejenige der septierten Fasern steigt vom Frühholz zum Spätholz an, wobei die Spätholzfasern im Mittel um $24 \%$ länger sind als die ersten Frühholzfasern. Die septierten Fasern sind hierbei stets gleichmäßig kürzer als die nicht- 